\title{
Systematic Review with Meta-Analysis
}

\section{Effect of plant foods and beverages on plasma non-enzymatic antioxidant capacity in human subjects: a meta-analysis}

\author{
Daniele Lettieri-Barbato ${ }^{1}$, Francesco Tomei ${ }^{2}$, Angela Sancini ${ }^{2}$, Giuseppa Morabito ${ }^{1}$ \\ and Mauro Serafini ${ }^{3 *}$ \\ ${ }^{1}$ Food and Nutrition Unit, IRCCS San Raffaele Pisana, Via della Pisana 235, 00163 Rome, Italy \\ ${ }^{2}$ Unit of Occupational Medicine, Department of Anatomy, Histology, Medical-Legal and the Orthopedics, University of Rome \\ "Sapienza", Viale Regina Elena 336, 00161 Rome, Italy \\ 3 "Functional Food and Metabolic Stress Prevention" Programme, CRA-Ex-INRAN National Institute for Food and Nutrition \\ Research, Via Ardeatina 546, 00178 Rome, Italy
}

(Submitted 25 May 2012 - Final revision received 8 January 2013 - Accepted 8 January 2013 - First published online 14 March 2013)

\begin{abstract}
Non-enzymatic antioxidant capacity (NEAC) represents a sensitive biomarker measuring the in vivo antioxidant potential of vegetable foods. To evaluate the effectiveness of plant-derived foods and beverages on the plasma non-enzymatic antioxidant system, we analysed all literature published upto May 2010. Data were extracted by two authors independently, and the effect size was summarised using standardised mean differences by a random-effects model. For the analysis, eighty-eight studies were included, reporting a total number of 122 interventions and involving 2890 subjects. There was overall evidence of the effectiveness of fruit, vegetables, dietary patterns based on plant foods, red wine and tea in increasing plasma NEAC. No changes were found for chocolate and fruit juices. We observed an overall effect size three times higher in subjects with risk factors when compared with healthy subjects. Total radical-trapping antioxidant parameter, oxygen radical absorbance capacity and ferric-reducing antioxidant power methods showed a similar increase in plasma NEAC following dietary supplementation, whereas Trolox equivalent antioxidant capacity did not respond to dietary supplementation. Data from the present meta-analysis show that plant-derived foods represent an effective strategy to enhance an endogenous antioxidant network in humans. This is particularly evident in the presence of oxidative stress-related risk factors.
\end{abstract}

Key words: Plant foods: Antioxidant capacity: Oxidative stress: Human nutrition: Flavonoids

A large body of epidemiological evidence strongly suggests a primary role for plant-based dietary patterns in reducing the risk of diseases ${ }^{(1)}$. However, the identification of the molecules involved in the protective effect of vegetable food and their mechanism of action is far from being understood ${ }^{(2)}$. Phytochemicals contained in the plant kingdom are hypothesised to reduce free radical-related cellular damage, potentiating redox defence of the body and contributing to reduction of the risk of developing oxidative stress-related diseases ${ }^{(3)}$. On the basis of the so-called 'antioxidant hypothesis', long-term clinical trials have been developed to investigate the effect of supplementation with natural antioxidants to reduce the development of oxidative stress-related diseases. However, clinical trials have produced extremely contrasting results, highlighting the difficulties in mimicking the healthy effect of plant-derived foods through the usage of natural supplements ${ }^{(4,5)}$. Moreover, a negative effect in increasing mortality rates of overall mortality and cancer has been observed in specific meta-analyses, raising strong concerns about the use of galenic antioxidants for disease prevention ${ }^{(5,6)}$.

Antioxidant defences of the body are composed of molecular and enzymatic players; however, the composition of this network markedly differs, in terms of concentration and components, between body compartments ${ }^{(7)}$. The multifunctional

Abbreviations: EXP, experimental studies; FRAP, ferric-reducing antioxidant power; NEAC, non-enzymatic antioxidant capacity; ORAC, oxygen radical absorbance capacity; OSRRF, oxidative stress-related risk factor; Q-EXP, quasi-experimental studies; SMD, standardised mean difference; TEAC, Trolox equivalent antioxidant capacity; TRAP, total radical-trapping antioxidant parameter.

*Corresponding author: M. Serafini, fax + 39 0651494550, email serafini_mauro@yahoo.it 
properties of the antioxidant network highlight the crucial importance of dynamic interactions among the components of the network in protecting body fluids from oxidative stress. Non-enzymatic antioxidant capacity (NEAC), defined as the moles of oxidants neutralised by 1 litre of plasma, represents a biomarker measuring the antioxidant potential, including synergistic interactions, of body fluids and tissues ${ }^{(7)}$. In terms of participation of individual components to the network, the main contributor to NEAC is uric acid (40-55\%), followed by thiol groups (10-24\%), ascorbic acid (8-15\%) and vitamin $\mathrm{E}$ (less than $10 \%)^{(8)}$. The contribution of single antioxidants to overall NEAC leaves unexplained a percentage ranging from 20 to $40 \%$ that might be accounted for by dietary components and/or synergistic interactions. NEAC methodology has been widely used to investigate the role of diet in modulating antioxidant function in humans, though criticisms have been raised on the real ability of NEAC to be a proper biomarker of antioxidant function in vivo ${ }^{(8,9)}$. Moreover, the large number of assays available for the measurement of NEAC in biological matrices has become a major obstacle in understanding the pros and cons of NEAC as a biomarker for assessing antioxidant capacity in vivo.

The main point of concern relies on the lack of a clear association between the ingestion of plant foods rich in antioxidants and changes in plasma NEAC. Plasma NEAC was shown to respond to the ingestion of dietary food items rich in antioxidants such as tea, wine, chocolate, fruit and vegetables in the majority of acute ingestion studies ${ }^{(8)}$. However, if we analyse chronic intervention studies, the picture is much more complex and results are not homogeneous. Recently, it has been suggested that the efficiency of antioxidants in modulating NEAC in chronic trials depends on the 'healthy status' of the subjects, with larger effects occurring in subjects characterised by risk factors (i.e. smoking), pollutant exposure ${ }^{(10)}$ or pathologies $\left(\mathrm{HIV}^{+} \text {and } \mathrm{CVD}\right)^{(8)}$. Healthy subjects might have a lower responsiveness to antioxidant supplementation because they do not have any oxidative stress conditions in which a higher intake of redox molecules might be required.

In order to clarify these concerns, we performed a metaanalysis of human intervention studies testing the effect of vegetable food and beverage consumption on plasma NEAC. Moreover, the effect of oxidative stress-related risk factors (OSRRF) and of the applied methodology was also investigated.

\section{Experimental methods}

\section{Search strategy}

An extensive search for 'antioxidant capacity', 'antioxidant potential', 'antioxidant status' and diet or specific food/ beverages (fruit, fruit juice, vegetable, tea, chocolate and wine) was performed, collecting dietary intervention studies up to May 2010. Papers suitable for inclusion were identified by systematic research on the MEDLINE and EMBASE electronic databases. Searches were limited to human subjects and results reported in the English language. In addition, a manual search of references from reports of clinical trials or review articles was performed to identify relevant studies.

\section{Study selection}

In the present meta-analysis, we included dietary intervention studies with an experimental design (EXP) as well as with a quasi-experimental design (Q-EXP). EXP studies were randomised, with a control group and a parallel or cross-over design. Observational studies (pre- and post-intervention or pre- and post-data), non-randomised or uncontrolled study design were included in the Q-EXP study category ${ }^{(11)}$. Q-EXP studies were pooled together with EXP studies only after assessing whether they were in agreement with EXP studies ${ }^{(12,13)}$.

General inclusion criteria were as follows: (1) human intervention studies assessing the effectiveness of foods, beverages or dietary patterns on plasma NEAC; (2) studies with both acute and chronic supplementation periods; (3) chronic studies with a treatment period longer than $7 \mathrm{~d}$; (4) healthy people and subjects characterised by OSRRF (smoking, dyslipidaemia, heart transplant, obesity and ageing) or pathologies ( $\mathrm{HIV}^{+}$and CVD); (5) numerical or graphical information about results, study duration (chronic studies) or time point (acute studies) and dosage of the plant-derived foods and beverages used. Studies were excluded when only abstracts were available; experimental meal did not fall into the categories that we considered; data presentation was incomplete; treatment effects on NEAC were reported as AUC; subjects were $<18$ years; information about the dose of food and beverages was incomplete; the washout time was not considered or was less than $7 \mathrm{~d}$ (only for the cross-over design).

When necessary, efforts were made to contact investigators for clarification or additional data.

\section{Quality assessment and data extraction}

For data extraction, two investigators (D. L.-B. and G. M.) independently screened the titles and abstracts resulting from the search strategies. Articles with titles or abstracts clearly irrelevant were rejected at first screening. The full text of potentially relevant articles was reviewed to assess eligibility for inclusion in the meta-analysis with any disagreement resolved by consensus. Considering that the present meta-analysis included different types of studies, it was performed following both PRISMA (Preferred Reporting Items for Systematic Reviews and MetaAnalyses) and MOOSE (Meta-Analysis of Observational Studies in Epidemiology) checklists ${ }^{(14)}$.

Quality assessment of the included studies was performed using study design as the main quality variable (parallel, cross-over studies or pre-post-intervention studies, placebo, studies with or without a control group). Moreover, other features related to human dietary intervention studies, such as the number of participants enrolled, the length of studies for chronic ones and the description of experimental meal, were considered. Score quality was not used as a criterion for the selection of trials, but it was only used for allocation purpose in high-, fair or low-bias risk. Trials with an adequate study design were classified as low-bias risk (good quality: $>7$ points), whereas those with an adequate design but with one or more unclear quality components were classified as fair quality (4-7 points). Trials with an inadequate study 
Table 1. Criteria utilised to assess the quality of the studies included in the meta-analysis

\begin{tabular}{|c|c|c|c|c|c|c|c|c|c|c|}
\hline \multirow[b]{2}{*}{ Trials } & \multicolumn{3}{|c|}{ Study design } & \multirow{2}{*}{$\begin{array}{l}\text { No. of } \\
\text { participants } \\
\text { enrolled } \\
\\
>10 \\
\text { subjects }\end{array}$} & \multirow{2}{*}{$\begin{array}{l}\text { Length of } \\
\text { the study } \\
\text { (for chronic } \\
\text { studies) } \\
>12 d\end{array}$} & \multirow{2}{*}{$\begin{array}{c}\text { Description of } \\
\text { experimental } \\
\text { meal } \\
\text { Main } \\
\text { characteristics }\end{array}$} & \multirow[b]{2}{*}{ Funding } & \multicolumn{3}{|c|}{ Quality assessment } \\
\hline & $\begin{array}{l}\text { Controlled (placebo } \\
\text { or control with food, } \\
\text { diet and beverages } \\
\text { without antioxidant) }\end{array}$ & $\begin{array}{l}\text { Randomised } \\
\text { (with parallel } \\
\text { or cross-over } \\
\quad \text { design) }\end{array}$ & $\begin{array}{c}\text { Pre- } \\
\text { post-data }\end{array}$ & & & & & Good & Fair & Poor \\
\hline Tea & $11 / 12$ & $8 / 12$ & $1 / 12$ & $9 / 12$ & $8 / 8$ & $5 / 12$ & $2 / 12$ & 8 & 3 & 1 \\
\hline Fruit juices & $7 / 19$ & $7 / 19$ & $12 / 19$ & $14 / 19$ & $13 / 14$ & $12 / 19$ & $4 / 19$ & 8 & 0 & 11 \\
\hline Red wine & $11 / 16$ & $6 / 16$ & $/ 16$ & $9 / 19$ & $3 / 4$ & $2 / 16$ & $6 / 16$ & 5 & 5 & 6 \\
\hline Chocolate & $9 / 11$ & $7 / 11$ & $2 / 11$ & $11 / 11$ & $5 / 5$ & $10 / 11$ & $4 / 11$ & 7 & 1 & 3 \\
\hline Fruit & $8 / 11$ & $6 / 11$ & $3 / 11$ & $8 / 11$ & $4 / 4$ & $4 / 11$ & $4 / 11$ & 6 & 1 & 4 \\
\hline Vegetables & $4 / 12$ & $2 / 12$ & $8 / 12$ & $9 / 12$ & $8 / 9$ & $4 / 12$ & $3 / 12$ & 1 & 2 & 9 \\
\hline Dietary patterns & $5 / 7$ & $5 / 7$ & $2 / 7$ & $7 / 7$ & $7 / 7$ & $5 / 7$ & $0 / 7$ & 2 & 2 & 3 \\
\hline
\end{tabular}

design were considered as high-bias risk or poor quality ( $<4$ points; Tables 1 and 2$)$. Extracted data include: name of the first author; year of publication; experimental meal; number of participants; health status of the enrolled subjects; type and design of the study and time point considered for data analysis; daily dosage; assay used; study quality.

\section{Data analysis}

Relevant data of the studies available for formal meta-analytic evaluation were entered into Comprehensive Meta-Analysis software (Biostat) for evaluation of the meta-analysis.

In particular, for parallel or cross-over studies, when results were reported as post-data only, we used mean, standard deviation and sample size in each group, or difference in means, sample size and $P$ value between groups. When results were reported as pre- and post-data, we used mean, standard deviation, sample size in each group and correlation between baseline and end-point intervention period, or mean change, standard deviation difference, sample size in each group, correlation between baseline and end-point intervention period. For observational studies considering only one group (pre-post-intervention data), we used mean difference, standard deviation of difference and sample size. For all data, standard deviations were calculated, when necessary, from standard error or $\mathrm{CI}$, and data not provided in numerical form were estimated from figures. In all types of dietary intervention studies, we assumed the correlation between baseline and end-point study period to be 0.5 to produce the most conservative estimate ${ }^{(13,15)}$.

When in the same study the effect on plasma NEAC of two different doses of the same experimental meal was evaluated, our approach was to create a synthetic variable for each study to compute a combined effect based on these variables (defined as the mean of the effect size), and we used it as the effect size in the analysis. The same procedure was used in chronic studies that reported the effect on NEAC of the same experimental meal, in two or more periods, as well as in acute studies that showed the effect on NEAC of the same experimental meal, in two or more time points.

For acute studies, the significant change (positive or negative) on plasma or serum NEAC was considered as the time point to statistical analysis, while if there were no reported significant changes, we have considered the time point at the end of the study. For chronic studies, we considered the end point of the intervention.

All studies addressed the same outcome (plasma antioxidant capacity or NEAC), used different methods of analysis (total radical-trapping antioxidant parameter (TRAP), ferric-reducing antioxidant power (FRAP), oxygen radical absorbance capacity (ORAC) and Trolox equivalent antioxidant capacity (TEAC)) and presented the results in different scales. To enable a joint comparison, the same index of treatment effects was used, and in each individual study, the standardised mean difference (SMD) with 95\% CI was calculated ${ }^{(16)}$. In the present analysis, a positive SMD indicates an increase in plasma NEAC after consumption of plant-derived foods and beverages (experimental meal). When an author published the plasma NEAC value detected with two or more methods, we evaluated separately the effect size in relation to the assay used.

Meta-analysis was performed using DerSimonian \& Laird's 'random-effects models'. Under the random-effects model, effect sizes are assumed not to be common to all studies; they vary under a normal distribution model ${ }^{(17)}$. We used a random-effects model because there was evidence of heterogeneity between studies based on the $\chi^{2}$ test for heterogeneity at the significant level of $P=0 \cdot 10$ and the $I^{2}$ statistic, percentage of total variation across studies due to heterogeneity, rather than chance, of more than $50 \%{ }^{(18)}$.

Potential publication bias (i.e. the association of publication probability with the statistical significance of study results) was

Table 2. Quality score used in the meta-analysis

\begin{tabular}{lc}
\hline & Score \\
\hline Randomised (with a parallel or cross-over design) & 2 \\
Group controlled without an antioxidant meal & 2 \\
$\quad$ (with a parallel or cross-over design) & \\
Placebo controlled (with a parallel or cross-over design) & 2 \\
Blinding & 1 \\
Funding & 0 \\
Length of the study (for chronic studies) & 1 \\
No. of participants enrolled (>10 subjects) & 1 \\
Description of experimental meal & 1 \\
\hline
\end{tabular}


investigated using visual assessment of the funnel plot calculated by Comprehensive Meta-Analysis software ${ }^{(19)}$ (Biostat).

\section{Results}

We identified 716 potential literature citations through the database searches (Fig. 1). At first, 156 studies met our inclusion criteria and five additional studies potentially meeting our inclusion criteria were identified through scanning reference lists. Of the 161 references, seventy-three papers were excluded for the following reasons: outcome measures not suitable for meta-analysis, continuous variables of preand post-intervention data or mean difference for only postdata not included in the publication; NEAC values expressed as AUC; chronic intervention trials were less than $7 \mathrm{~d}$ or intervention studies performed with dietary supplements. The remaining eighty-eight studies, reporting a total number of 122 interventions met our selection criteria and were included in the meta-analysis (Table S1, available online).

The following three main categories of intervention, on the basis of type of food, were identified: beverages ( $n$ 47); plant foods ( $n$ 34); plant food-based dietary patterns ( $n$ 7). Considering the criteria used to assess the quality of studies included in the present meta-analysis, thirty-six studies were of good quality, fifteen studies were fair and thirty-seven studies were of poor quality. Moreover, forty of the included studies had an experimental design (EXP), whereas forty-eight studies had a quasi-experimental design (Q-EXP). Plasma NEAC was assessed in 2890 subjects. Moreover, all subjects were stratified into two main subgroups: healthy subjects ( $n 1880)$ and subjects with risk factors or diseases ( $n$ 1010). After evaluating the agreement between Q-EXP and EXP studies through the view of effect size direction (data not shown), both Q-EXP and EXP studies were processed together ${ }^{(13)}$.

\section{Beverages}

Intervention studies involving red wine ( $n$ 16), fruit juices ( $n$ 20) and tea ( $n$ 12) are described in Fig. 2. There was clear evidence on the efficacy of red wine consumption in increasing plasma NEAC, both in acute feeding studies (SMD 1·118, 95\% CI 0.976, 1.325; $P<0.000$ ) and in chronic studies (SMD 1.130, $95 \%$ CI $0.818,1.617 ; P<0.001)$. Fruit juice ingestion was able to increase plasma NEAC in acute feeding studies (SMD 1.028, $95 \%$ CI $0.446,1 \cdot 702 ; P=0 \cdot 001)$, but was ineffective in chronic intervention studies (SMD 0.157, 95\% CI -0.275, 0.422; $P=0.382$ ). Tea consumption induced a similar increase in plasma NEAC both after acute (SMD 0.703, 95\% CI 0.154, 1.248; $P=0.012$ ) and chronic ingestion (SMD 0.664, 95\% CI $0.249,1.078 ; P=0.002)$. The results showed that green tea had a stronger antioxidant effect than black tea (SMD 0.957, 95\% CI 0.447, 1.466; $P<0.0001$ for green tea and SMD 0.487, 95\% CI $0.072,0.901 ; P=0.021$ for black tea) (data not shown).

\section{Plant foods}

The effect of chocolate ( $n$ 11), fruits $(n 10)$ and vegetables ( $n$ 12) on plasma NEAC is described in Fig. 3. Chocolate

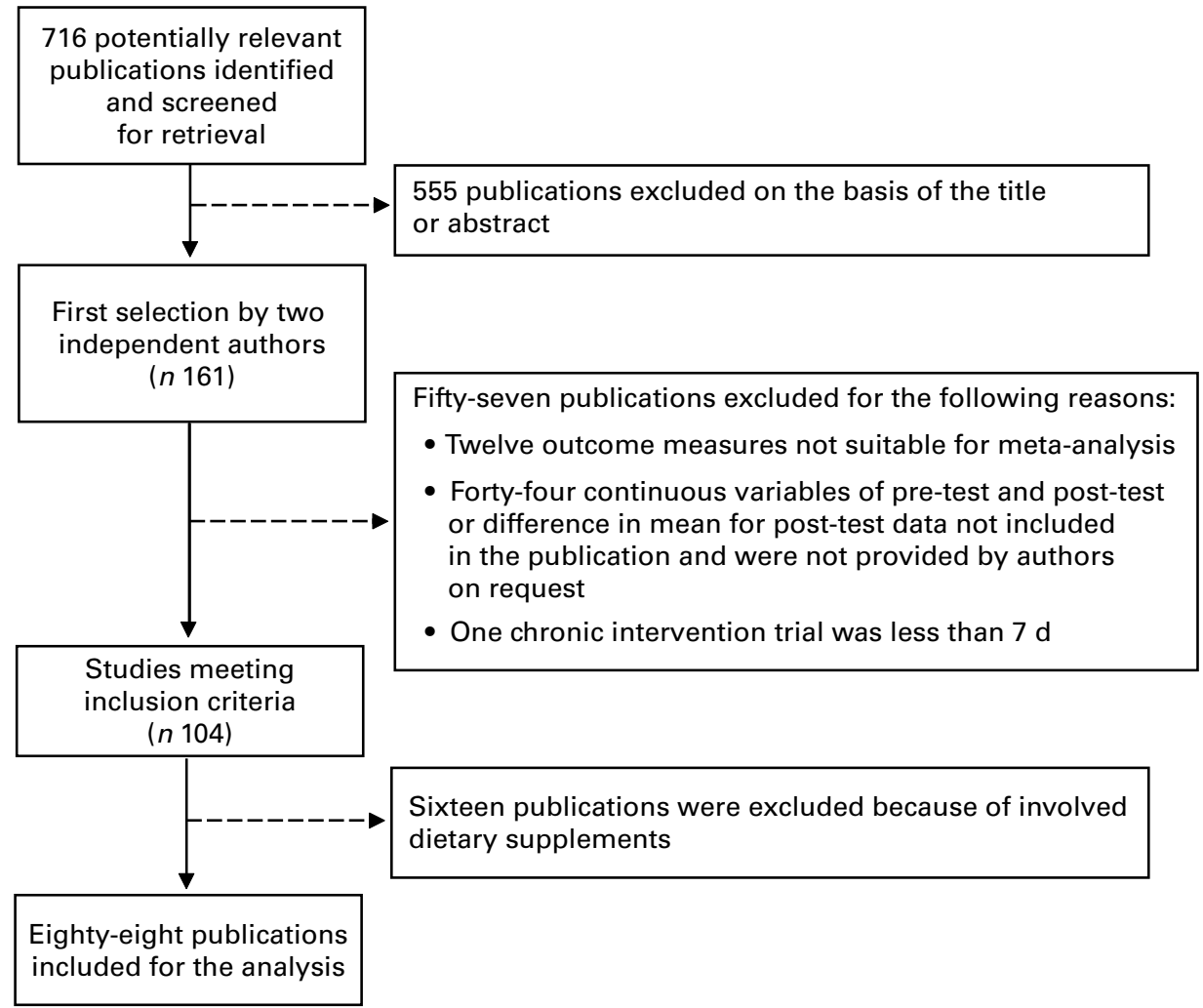

Fig. 1. Flow chart of the study identification and selection. 
Red wine

\begin{tabular}{|c|c|c|}
\hline & Study & Dose \\
\hline Acute & Vukovic, $2009^{(39)}$ & $3 \mathrm{ml} / \mathrm{kg}$ \\
\hline Acute & Maxwell, 1994 & $5-7 \mathrm{ml} / \mathrm{kg}$ \\
\hline Acute & Modun, $2008^{(41)}$ & $3 \mathrm{ml} / \mathrm{kg}$ \\
\hline Acute & Arendt, $2005^{(42)}$ & $200 \mathrm{ml}$ \\
\hline Acute & Serafini, $1998^{(43)}$ & $113 \mathrm{ml}$ \\
\hline Acute & Blackhurst, $2006^{(45)}$ & About $200 \mathrm{~m}$ \\
\hline Acute & Cao, $1998 \_a^{(66)}$ & $300 \mathrm{ml}$ \\
\hline Acute & Cao, $1998 b^{(66)}$ & $300 \mathrm{ml}$ \\
\hline Acute & Day, $1995^{(47)}$ & $250 \mathrm{ml}$ \\
\hline Acute & Fernández-Pachón, 2005_a $a^{(48)}$ & $300 \mathrm{ml}$ \\
\hline Acute & Fernández-Pachón, 2005_b $b^{(48)}$ & $300 \mathrm{ml}$ \\
\hline Acute & Pinzani, $2010^{(49)}$ & $300 \mathrm{ml}$ \\
\hline Acute & Simonetti, $2001^{(50)}$ & About $200 \mathrm{~m}$ \\
\hline Acute & Kiviniemi, $2007^{(54)}$ & $0.75 \mathrm{~g} / \mathrm{kg}$ \\
\hline \multicolumn{3}{|l|}{ Acute } \\
\hline Chronic & Avellone, $2006^{(44)}$ & $250 \mathrm{ml}$ \\
\hline Chronic & Otaolaurruchi, 2007_a $a^{(51)}$ & $300 \mathrm{ml}$ \\
\hline Chronic & Otaolaurruchi, 2007_b $\mathrm{b}^{(51)}$ & $300 \mathrm{ml}$ \\
\hline Chronic & Tsang, $2005^{(52)}$ & $375 \mathrm{ml}$ \\
\hline Chronic & Guarda, $2005^{(53)}$ & $250 \mathrm{ml}$ \\
\hline \multicolumn{3}{|l|}{ Chronic } \\
\hline Overall & & \\
\hline
\end{tabular}

\begin{tabular}{|c|c|c|}
\hline Quality & Participants $(n)$ & SMD \\
\hline Good & 10 & $1 \cdot 377$ \\
\hline Fair & 10 & 1.599 \\
\hline Good & 9 & 1.263 \\
\hline Good & 19 & 0.000 \\
\hline Good & 19 & 1.708 \\
\hline Good & 15 & -0.168 \\
\hline Poor & 16 & $2 \cdot 304$ \\
\hline Poor & 16 & 0.632 \\
\hline Poor & 6 & 1.388 \\
\hline Poor & 8 & 0.883 \\
\hline Poor & 8 & 1.065 \\
\hline Poor & 5 & 0.451 \\
\hline Poor & 5 & 1.552 \\
\hline \multirow[t]{2}{*}{ Poor } & 18 & 2.018 \\
\hline & & $1 \cdot 118$ \\
\hline Fair & 48 & 1.875 \\
\hline Fair & 16 & 0.365 \\
\hline Fair & 16 & $1 \cdot 150$ \\
\hline Fair & 20 & 0.496 \\
\hline \multirow[t]{3}{*}{ Fair } & 20 & 1.610 \\
\hline & & $1 \cdot 130$ \\
\hline & & $1 \cdot 121$ \\
\hline
\end{tabular}

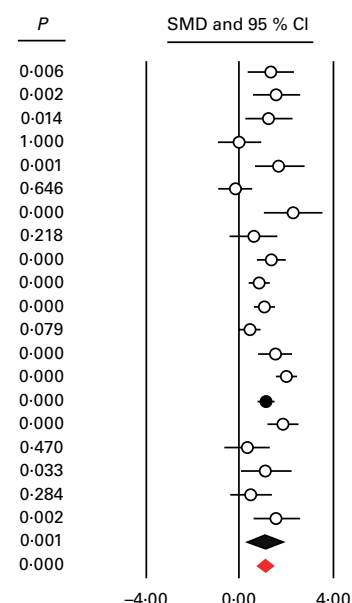

Fruit juice

\begin{tabular}{|c|c|}
\hline & Study \\
\hline Acute & Chaves, 2009_a $a^{(103)}$ \\
\hline Acute & Netzel, $2002^{(95)}$ \\
\hline Acute & Netzel, 2005_a $a^{(96)}$ \\
\hline Acute & Netzel, 2005_b $b^{(96)}$ \\
\hline Acute & Pedersen, 2000_ $a^{(97)}$ \\
\hline Acute & Pedersen, 2000_ $b^{(97)}$ \\
\hline Acute & Prior, $2007^{(98)}$ \\
\hline Acute & Vinson, 2008 ${ }^{(100)}$ \\
\hline Acute & \\
\hline Chronic & Arendt, 2001_a(83) \\
\hline Chronic & Arendt, 2001_b $\mathrm{b}^{(83)}$ \\
\hline Chronic & Borochov-Neori, 2006 ${ }^{(93)}$ \\
\hline Chronic & Bub, 2003_a(85)' \\
\hline Chronic & Bub, 2003_ $b^{(85)}$ \\
\hline Chronic & Castilla, 2006_a $a^{(84)}$ \\
\hline Chronic & Castilla, 2006_b b $^{\text {(84) }}$ \\
\hline Chronic & Chaves, 2009 $b^{(103)}$ \\
\hline Chronic & Cilla, 2009_a $a^{(\overline{8} 6)}$ \\
\hline Chronic & Cilia, 2009_b \\
\hline Chronic & Duthie, $2006^{(87)}$ \\
\hline Chronic & Gorinstein, $2004^{(94)}$ \\
\hline Chronic & Guo, 2008_a $a^{(88)}$ \\
\hline Chronic & Guo, 2008_b ${ }^{(88)}$ \\
\hline Chronic & O'Byrne, $2002^{(89)}$ \\
\hline Chronic & Park, 2009 \\
\hline Chronic & Riso, $2005^{(91)}$ \\
\hline Chronic & Ruel, $2005^{(99)}$ \\
\hline Chronic & Wilms, 2007 \\
\hline Chronic & Young, 1999_a(92) \\
\hline Chronic & Young, 1999_b $\mathbf{b}^{(92}$ \\
\hline Chronic & \\
\hline
\end{tabular}

$\frac{\text { Dose }}{2 \cdot 5 \mathrm{cups}}$
$400 \mathrm{ml}$
$300 \mathrm{ml}$
$300 \mathrm{ml}$
$500 \mathrm{ml}$
$500 \mathrm{ml}$
$315 \mathrm{ml}$
$240 \mathrm{ml}$
$1000 \mathrm{ml}$
$1000 \mathrm{ml}$
$200 \mathrm{ml}$
$330 \mathrm{ml}$
$330 \mathrm{ml}$
$100 \mathrm{ml}$
$100 \mathrm{ml}$
$2.5 \mathrm{cups}$
$500 \mathrm{ml}$
$500 \mathrm{ml}$
$750 \mathrm{ml}$
$150 \mathrm{ml}$
$250 \mathrm{ml}$
$250 \mathrm{ml}$
$10 \mathrm{ml} / \mathrm{kg}$
$5.5 \mathrm{ml} / \mathrm{kg}$
$600 \mathrm{ml}$
$7 \mathrm{ml} / \mathrm{kg}$
$1000 \mathrm{ml}$
$1083 \mathrm{ml}$
$1083 \mathrm{ml}$

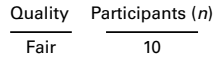

Poor

Poor
Poor

Poor

Good

Good

Poor

Poor

Poor

Poor
Poor
Poor

Poor

Food

Poor
Poor

Goor

Good

Poor
Poor

Poor

Good

Good
Poor
Poor

Poor
Poor
Poor

10
6
16
16
18
18
6

$\frac{S M D}{0.557}$

0.850
1.133
4.665
1.248

$1 \cdot 248$

1.248
0.164
0.637
0.846
1.028

0.846
1.028
1.973
0.397

0.397
0.577

0.577
-0.088

-0.088
0.211

0.211
0.934
0.841

0.841
3.804

-4.068
-5.4608

-4.068
-5.4608

-0.272
0.148

0.148
0.571

0.571
-0.074

-0.074
0.866
0.600
0.170

0.600
0.170

0.170
0.846

0.846
0.371

$-0.006$

-0.096
0.157

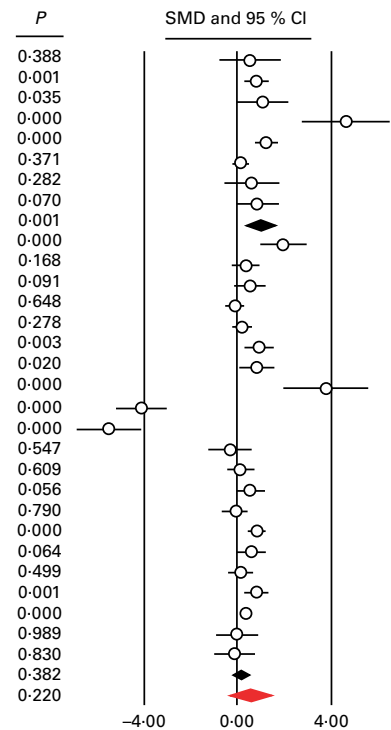

Tea

\begin{tabular}{|c|c|c|}
\hline & Study & Dose \\
\hline Acute & Maxwell, $1996^{(35)}$ & $500 \mathrm{ml}$ \\
\hline Acute & Hodgson, 2000_ $\mathrm{b}^{(32)}$ & 4 cups \\
\hline Acute & Kyle, $2007^{(37)}$ & $400 \mathrm{ml}$ \\
\hline Acute & Panza, 2008 ${ }^{(29)}$ & $600 \mathrm{ml}$ \\
\hline Acute & Hodgson, 2000_ $\mathrm{a}^{(32)}$ & 4 cups \\
\hline Acute & Serafini, 1996_b ${ }^{(33)}$ & $300 \mathrm{ml}$ \\
\hline \multicolumn{2}{|l|}{ Acute } & $300 \mathrm{ml}$ \\
\hline Chronic & Davies, $2003^{(34)}$ & 5 cups \\
\hline Chronic & Duffy, 2001_a $\mathrm{a}^{(36)}$ & $900 \mathrm{ml}$ \\
\hline Chronic & Duffy, 2001_b $\mathrm{b}^{(36)}$ & $900 \mathrm{ml}$ \\
\hline Chronic & Van het hof, 1997_b $b^{(31)}$ & $900 \mathrm{ml}$ \\
\hline Chronic & Widlansky, 2005_a ${ }^{(38)}$ & $900 \mathrm{ml}$ \\
\hline Chronic & Widlansky, 2005_b $\mathrm{b}^{(38)}$ & $900 \mathrm{ml}$ \\
\hline Chronic & Bertipaglia de santana, $2008^{(27)}$ & $500 \mathrm{ml}$ \\
\hline Chronic & Erba, 2005 & 2 cups \\
\hline Chronic & Coimbra, $2006^{(30)}$ & $1000 \mathrm{ml}$ \\
\hline Chronic & van het Hof, 1997_a $a^{(31)}$ & $900 \mathrm{ml}$ \\
\hline & & \\
\hline rall & & \\
\hline
\end{tabular}

\begin{tabular}{|c|c|}
\hline Quality & Participants $(n)$ \\
\hline Poor & 10 \\
\hline Good & 40 \\
\hline Fair & 5 \\
\hline Fair & 28 \\
\hline Good & 40 \\
\hline Good & 10 \\
\hline Good & 10 \\
\hline Good & 15 \\
\hline Good & 42 \\
\hline Good & 42 \\
\hline Fair & 32 \\
\hline Good & 44 \\
\hline Good & 44 \\
\hline Good & 50 \\
\hline Good & 24 \\
\hline Fair & 68 \\
\hline Fair & 32 \\
\hline
\end{tabular}

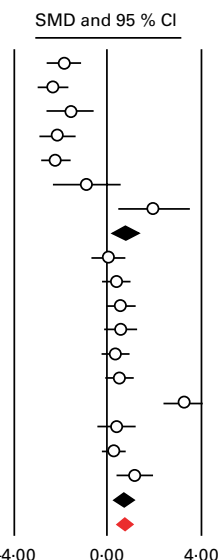

Fig. 2. Effect of beverage ingestion on plasma non-enzymatic antioxidant capacity (NEAC). Studies were stratified according to the design of the study (acute or chronic). A positive standardised mean difference (SMD) indicates an increase in plasma NEAC, whereas a negative SMD indicates a decrease in plasma NEAC. The terms $a, b$ or $c$ was used when the same author measured plasma NEAC with different methods. (A colour version of this figure can be found online at http://www.journals.cambridge.org/bjn). 
Chocolate

\begin{tabular}{|c|c|c|c|c|}
\hline & Study & Dose & Quality & Participants $(n)$ \\
\hline Acute & Flammer, 2007_a $\mathrm{a}^{(68)}$ & $40 \mathrm{~g}$ & Good & 22 \\
\hline Acute & Flammer, 2007_b $b^{(68)}$ & $40 \mathrm{~g}$ & Good & 22 \\
\hline Acute & Serafini, $2003^{(79)}$ & $100 \mathrm{~g}$ & Poor & 12 \\
\hline Acute & Rein, $2000^{(71)}$ & $80 \mathrm{~g}$ & Good & 13 \\
\hline Acute & Wiswedel, 2004 & $100 \mathrm{ml}$ & Good & 20 \\
\hline Acute & Wang, 2000 $0^{(75)}$ & $53 \mathrm{~g}$ & Poor & 22 \\
\hline Acute & Viachopoulos, $2005^{(76)}$ & $100 \mathrm{~g}$ & Good & 34 \\
\hline \multicolumn{5}{|l|}{ Acute } \\
\hline Chronic & Mathur, 2002 (67) & $78 \mathrm{~g}$ & Poor & 25 \\
\hline Chronic & Fraga, $2005^{(20)}$ & $105 \mathrm{~g}$ & Good & 28 \\
\hline Chronic & Murphy, $2003^{(70)}$ & Six tablets & Good & 28 \\
\hline Chronic & Mursu, 2004_(73) & $75 \mathrm{~g}$ & Fair & 30 \\
\hline Chronic & Mursu, 2004_b $b^{(73)}$ & $75 \mathrm{~g}$ & Fair & 30 \\
\hline Chronic & Engler, $2004^{(74)}$ & $46 \mathrm{~g}$ & Good & 21 \\
\hline \multicolumn{5}{|l|}{ Chronic } \\
\hline Overall & & & & \\
\hline
\end{tabular}

\begin{tabular}{|c|c|}
\hline SMD & $P$ \\
\hline 0.020 & 0.963 \\
\hline 0.069 & 0.872 \\
\hline 1.500 & 0.000 \\
\hline 2.601 & 0.002 \\
\hline 0.708 & 0.124 \\
\hline 0.685 & 0.124 \\
\hline-0.032 & 0.926 \\
\hline 0.677 & 0.008 \\
\hline-0.327 & 0.111 \\
\hline-0.719 & 0.065 \\
\hline 0.187 & 0.622 \\
\hline 0.311 & 0.398 \\
\hline 0.356 & 0.334 \\
\hline 0.068 & 0.877 \\
\hline-0.034 & 0.897 \\
\hline 0.323 & 0.364 \\
\hline
\end{tabular}

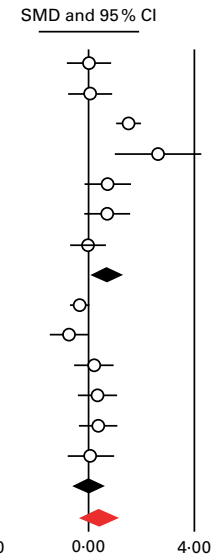

Fruit

\section{Study}

Cao, 1998_a $a^{(46)}$

Cao, 1998_b $b^{(46)}$

Lotito, $2004^{(104)}$

Mazza, 2002-a ${ }^{(105)}$

Mazza, 2002_b $b^{(105)}$

Prior, $2007^{(98)}$

Serafini, 2009_a(108)

Serafini, 2009_ $b^{(108)}$

Vinson, 2005 ${ }^{(109)}$

McAnulty, 2005(102)

Parker, 2007_a ${ }^{(106)}$

Parker, 2007_a ${ }^{(106)}$

Parker, 2007_(106)

Rankin, 2008 (107)

Tesorilere, 2004(109)

$\begin{array}{ccc}\frac{c}{\text { Dose }} & & \text { Quality } \\ 240 \mathrm{~g} & & \text { Poor } \\ 240 \mathrm{~g} & & \text { Poor } \\ \text { Five fruits } & \text { Good } \\ 100 \mathrm{~g} & \text { Good } \\ 100 \mathrm{~g} & \text { Good } \\ 189 \mathrm{~g} & \text { Good } \\ 200 \mathrm{~g} & \text { Poor } \\ 200 \mathrm{~g} & \text { Poor } \\ 40 \mathrm{~g} & \text { Good } \\ & \\ 250 \mathrm{~g} & \text { Good } \\ 250 \mathrm{~g} & \text { Poor } \\ 50 \mathrm{~g} & \text { Poor } \\ 50 \mathrm{~g} & \text { Poor } \\ 90 \mathrm{~g} & \text { Good } \\ 500 \mathrm{~g} & \text { Poor } \\ & \end{array}$

\begin{tabular}{c} 
Participants $(n)$ \\
\hline 16 \\
16 \\
12 \\
10 \\
10 \\
10 \\
11 \\
11 \\
10 \\
\\
20 \\
15 \\
15 \\
15 \\
17 \\
18
\end{tabular}

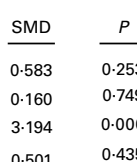

$\begin{array}{ll}0.501 & 0.435 \\ 1.488 & 0.037\end{array}$

$\begin{array}{ll}1.488 & 0.037 \\ 1.852 & 0.001\end{array}$

$0.711 \quad 0.035$

$1.383 \quad 0.001$

$2.093 \quad 0.000$

$1.260 \quad 0.000$
0.317

$0.317 \quad 0.481$

$2.000 \quad 0.000$

$\begin{array}{ll}-0667 & 0.020\end{array}$

$0.765 \quad 0.009$

$0.509 \quad 0.113$

$0.359 \quad 0.140$

$\begin{array}{ll}0.508 & 0.105\end{array}$

Vegetables

\begin{tabular}{|c|c|c|c|c|c|c|c|}
\hline & Study & Dose & Quality & Participants $(n)$ & SMD & $P$ & SMD and $95 \% \mathrm{Cl}$ \\
\hline Acute & Cao, 1998_a $a^{(46)}$ & $294 \mathrm{~g}$ & Poor & 16 & 2.770 & 0.000 & - \\
\hline Acute & Cao, 1998_b $\mathrm{b}^{(46)}$ & $294 \mathrm{~g}$ & Poor & 16 & 0.359 & 0.476 & م- \\
\hline Acute & McAnlis, $1999^{(64)}$ & $225 \mathrm{~g}$ & Poor & 5 & 0.395 & 0.120 & - \\
\hline Acute & Serafini, $2002^{(59)}$ & $250 \mathrm{~g}$ & Poor & 11 & 0.394 & 0.022 & 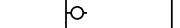 \\
\hline Acute & & & & & 0.629 & 0.012 & $<$ \\
\hline Chronic & Ahuja, 2006 $6^{(63)}$ & $30 \mathrm{~g}$ & Fair & 27 & 0.523 & 0.059 & D- \\
\hline Chronic & Castenmiller, $1999^{(62)}$ & $20 \mathrm{~g} \mathrm{MJ}$ & Fair & 58 & $-0 \cdot 118$ & 0.734 & \\
\hline Chronic & Jacob, 2008_a $a^{(65)}$ & $250 \mathrm{ml}$ & Poor & 12 & 0.093 & 0.748 & \\
\hline Chronic & Jacob, 2008_b $b^{(65)}$ & $250 \mathrm{ml}$ & Poor & 12 & -0.053 & 0.852 & \\
\hline Chronic & Lee, 2000_a $a^{(61)}$ & $230 \mathrm{~g}$ & Poor & 6 & $1 \cdot 109$ & 0.033 & -0 \\
\hline Chronic & Lee, 2000_b $b^{(61)}$ & $230 \mathrm{~g}$ & Poor & 6 & 0.218 & 0.598 & \\
\hline Chronic & Pellegrini, $2002^{(60)}$ & $25 \mathrm{~g}$ & Poor & 11 & 0.226 & 0.460 & - \\
\hline Chronic & Shen, 2007_a $\mathrm{a}^{(58)}$ & $500 \mathrm{~g}$ & Poor & 8 & $1 \cdot 344$ & 1.006 & -0 \\
\hline Chronic & Shen, 2007_ $\mathrm{b}^{(58)}$ & $600 \mathrm{~g}$ & Poor & 8 & $1 \cdot 109$ & 1.014 & $\rightarrow$ \\
\hline Chronic & Stracke, 2009_a(57) & $200 \mathrm{~g}$ & Good & 24 & 0.341 & 0.407 & o- \\
\hline Chronic & Stracke, 2009_b ${ }^{(57)}$ & $200 \mathrm{~g}$ & Good & 24 & $-0 \cdot 118$ & 0.773 & \\
\hline Chronic & Stracke, 2009_ ${ }^{(57)}$ & $200 \mathrm{~g}$ & Good & 24 & 0.159 & 0.697 & - \\
\hline Chronic & Tyssandlier, 2004 ${ }^{(56)}$ & $96 \mathrm{~g}$ & Poor & 20 & 1.000 & 0.000 & - \\
\hline Chronic & Vislioli, 2003 ${ }^{(55)}$ & $310 \mathrm{~g}$ & Poor & 12 & $0 \cdot 167$ & 0.566 & D- \\
\hline Chronic & & & & & 0.383 & 0.004 & $\bullet$ \\
\hline Overall & & & & & 0.439 & 0.000 & $\bullet$ \\
\hline
\end{tabular}

Fig. 3. Effect of plant food ingestion on plasma non-enzymatic antioxidant capacity (NEAC). Studies were stratified according to the design of the study (acute or chronic). A positive standardised mean difference (SMD) indicates an increase in plasma NEAC, whereas a negative SMD indicates a decrease in plasma NEAC. The terms $\mathrm{a}, \mathrm{b}$ or $\mathrm{c}$ was used when the same author measured plasma NEAC with different methods. (A colour version of this figure can be found online at http://www.journals.cambridge.org/bjn).

increased plasma NEAC in acute studies (SMD 0.677, 95\% CI 0.509, 1.066; $P<0.008$ ), but failed to enhance plasma NEAC after chronic ingestion (SMD - 0.034, 95\% CI - 0.540, 0.473; $P=0.897)$. Similarly, antioxidants from fruits massively increase endogenous antioxidant defences in acute studies (SMD 1.260, 95\% CI 0.946, 1.535; P<0.000), while no effect was revealed in chronic feeding studies (SMD 0.508, 95\% CI $0.133,0.938 ; P=0.105)$. On the other hand, vegetables 
induced a significant increase in NEAC both after acute (SMD $0 \cdot 629,95 \%$ CI $0.141,1 \cdot 117 ; P<0 \cdot 012)$ and chronic consumption (SMD 0.383, 95\% CI 0.120, 0.647; P=0.004).

\section{Plant food-based dietary patterns}

In agreement with the results observed for fruit and vegetables, dietary patterns based on fruit and vegetables had a strong impact in modulating positively plasma NEAC (SMD 0.965, 95\% CI 0.809, 1.122; $P<0.000$; Fig. 4).

\section{Effect of oxidative stress-related risk factors on plasma non-enzymatic antioxidant capacity}

As described in Table 3, participants enrolled in chronic studies were divided into healthy subjects (no detectable exposure to oxidative stress-related risk factors) and subjects exposed to oxidative stress-related risk factors. For the beverage category, an effect on plasma NEAC was clearly detected in the risk factor category (SMD $0 \cdot 765,95 \%$ CI 0.310, 1.220; $P<0.001$ ), whereas no changes in plasma NEAC were observed in healthy subjects (SMD 0.177, 95\% CI -0.154, $0.508 ; P<0.296$ ). On the contrary, in the food group (chocolate, fruit, vegetables and plant food-based dietary patterns), a significant increase in plasma NEAC in both categories was observed $(P<0.001$ for healthy and OSRRF subjects). However, the efficiency of dietary supplementation was much higher in subjects characterised by risk factors (SMD $1.253,95 \%$ CI $0.685,1.820)$ than in healthy subjects (SMD $0.502,95 \%$ CI $0.235,0.769 ; P<0.001)$. Finally, when all the available data (beverages and foods) were grouped, we observed that the increase in plasma NEAC was three times higher in subjects with risk factors (SMD 0.937, 95\% CI $0.592,1.281 ; P<0.001)$ when compared with healthy subjects (SMD 0.367, 95\% CI 0.162, 0.572; $P<0 \cdot 001$ ).

\section{Effect of methodology on plasma non-enzymatic antioxidant capacity}

The influence of methodology on the response of plasma NEAC after dietary supplementation with antioxidant-rich plant foods and beverages is described in Table 4. When methodologies based on the hydrogen atom transfer reaction were analysed, a positive response was detected in TRAP and ORAC, both in acute (SMD 1.113, 95\% CI 0.486, 1.741; $P=0.001 ;$ SMD $1.308,95 \%$ CI $0.704,1.911 ; P<0.001)$ and chronic studies (SMD 0.574, 95\% CI $0.078,1.071 ; P=0.023$; SMD $0.653,95 \%$ CI 0.304, 1.001; $P=0 \cdot 001)$. When single-electron transfer-based methods were tested, FRAP was increased both in acute (SMD 1.059, 95\% CI 0.612, 1.505; $P<0.001$ ) and chronic periods (SMD 0.366, 95\% CI 0.076, 0.656; $P=0.013$ ). On the contrary, the TEAC assay was unable to detect changes in plasma NEAC after dietary supplementation with vegetable foods (acute studies: SMD 0.08, 95\% CI $-0.660,0.819$; $P=0.819$ and chronic studies: SMD $0.318,95 \% \mathrm{CI}-0.075$, $0 \cdot 711 ; P=0 \cdot 113)$.

\section{Discussion}

The present meta-analysis shows that the ingestion of antioxidants from most vegetable foods and beverages differently modulates plasma NEAC in human subjects. The antioxidant effect is more prominent in subjects exposed to cardiovascular risk factors or affected by oxidative stress-related pathologies.

Analysing the plant-derived beverage category, we noted that red wine, black tea and green tea displayed an excellent antioxidant effect, in both acute and chronic studies. In agreement with epidemiological evidence, studies analysing the antioxidant potential of red wine, included in the present meta-analysis, used a moderate amount of red wine (about $250 \mathrm{ml}$ ), suggesting that the protective role of moderate red wine intake could be linked to its ability to enhance the body's antioxidant system. On the other hand, fruit juice

Dietary patterns based on plant-derived food

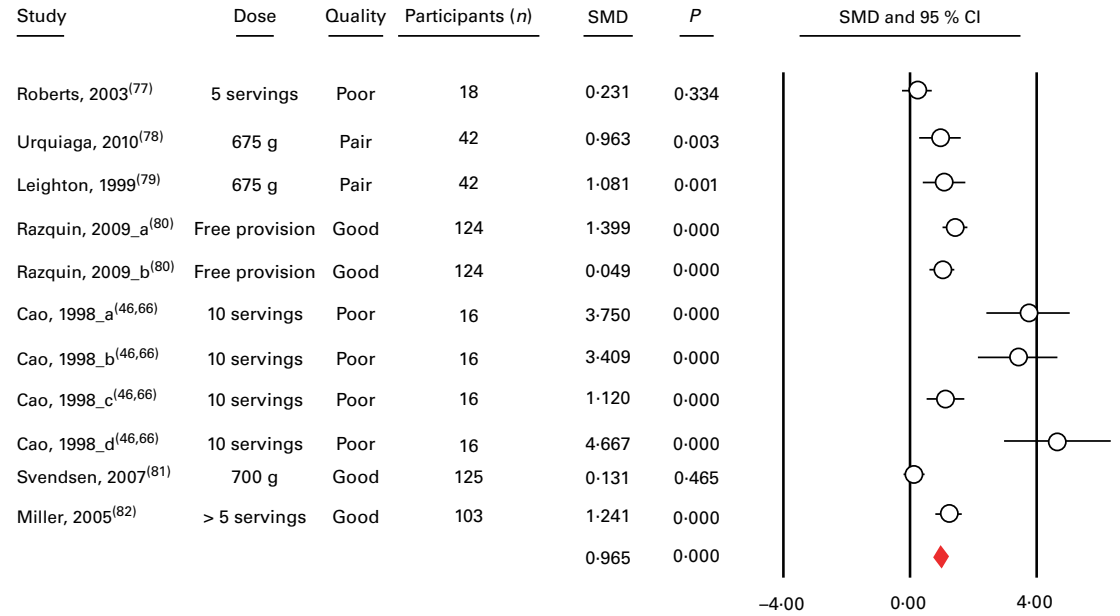

Fig. 4. Effect of chronic ingestion of plant food-based dietary patterns on plasma non-enzymatic antioxidant capacity (NEAC). A positive standardised mean difference (SMD) indicates an increase of plasma NEAC, whereas a negative SMD indicates a decrease in plasma NEAC. Terms a, b or $c$ was used when the same author measured plasma NEAC with different methods. (A colour version of this figure can be found online at http://www.journals.cambridge.org/bjn). 
Table 3. Chronic dietary supplementation studies with beverages and solid foods containing antioxidants and plasma non-enzymatic antioxidant capacity in human subjects: effect of oxidative stress-related risk factors (OSRRF)

(Number of interventions and $95 \%$ confidence intervals)

\begin{tabular}{|c|c|c|c|c|c|c|}
\hline \multirow[b]{2}{*}{ Treatments } & \multirow[b]{2}{*}{ Status } & \multirow[b]{2}{*}{ No. of interventions } & \multirow[b]{2}{*}{ Results } & \multicolumn{2}{|c|}{ Effect size } & \multirow[b]{2}{*}{$P^{\star}$} \\
\hline & & & & SMD & $95 \% \mathrm{Cl}$ & \\
\hline \multirow[t]{2}{*}{ Beverages (tea, fruit juices and red wine) } & Healthy & 23 & $\leftrightarrow$ & 0.177 & $-0.154,0.508$ & 0.296 \\
\hline & OSRRF & 12 & $\uparrow$ & 0.765 & $0.310,1.220$ & 0.001 \\
\hline \multirow{2}{*}{$\begin{array}{l}\text { Food (chocolate, fruit, vegetables and dietary } \\
\text { patterns based on plant-derived food) }\end{array}$} & Healthy & 30 & $\uparrow$ & 0.502 & $0.235,0.769$ & $<0.001$ \\
\hline & OSRRF & 7 & $\uparrow$ & $1 \cdot 253$ & $0.685,1.820$ & $<0.001$ \\
\hline \multirow{2}{*}{ Overall } & Healthy & 53 & $\uparrow$ & 0.367 & $0.162,0.572$ & $<0.001$ \\
\hline & OSRRF & 19 & $\uparrow$ & 0.937 & $0.592,1.281$ & $<0.001$ \\
\hline
\end{tabular}

SMD, standardised mean difference; $\leftrightarrow$, unchanged; $\uparrow$, increased.

*Test for the overall effect.

failed to display any antioxidant action in chronic trials, differently from single-time ingestion where an antioxidant effect is detected. The heterogeneity of fruit juice composition could be one of the reasons for the variability in plasma NEAC following chronic consumption. Moreover, fruit juices rich in fructose or enriched with high-fructose maize syrup, inducing free radical production, could decrease the antioxidant properties of fruit juice ${ }^{(20)}$.

The plant-derived food category, such as chocolate, fruits and vegetables, showed a clear antioxidant response after acute ingestion, whereas only vegetables were able to increase plasma antioxidant capacity after chronic intervention trials.

Despite more evidence being needed, variability of fruit composition and presence of potential oxidative stress inducers, such as fructose, might be the basis of the lack of effect displayed by fruit and fruit juices. Chocolate, despite the high content in flavonols and antioxidants, failed to display any effect in chronic studies. However, if we analyse the chronic studies, we observe that in Fraga's ${ }^{(21)}$ study, milk chocolate, losing antioxidant properties in vivo, was tested. Moreover, subjects enrolled in five chronic intervention trials were characterised by a lack of risk factors for oxidative stress, which is a factor able to affect the in vivo antioxidant response of foods ${ }^{(7)}$. In order to determine whether cocoa could represent a valid source of antioxidant ingredients, more studies on people exposed to oxidative stress risk factors are needed.

Epidemiological data linked the protective role of plant foods on several diseases with their ability to ameliorate the body's redox network. In line with evidence suggested in these studies, we reported a strong increase in plasma NEAC in dietary patterns rich in the plant food category.

Considering the overall effects, we observed a difference in terms of NEAC responses on the basis of the study design. In particular, when compared with chronic designs, plasma NEAC is promptly increased after vegetable food ingestion in acute studies. Acute ingestion trials rely on the importance of having a short experimental window free from variables such as physical activity, diet and homeostasis that might affect NEAC in chronic studies. However, as for all the kinetics, once a peak of effect is reached, between 30 and $60 \mathrm{~min}$ for beverages and after $2 \mathrm{~h}$ for solid foods, plasma levels turn back to baseline levels in few hours. Acute ingestion models allow us to draw relatively fast conclusions about the 'potentiality' of the food to display an antioxidant action in human subjects. It might be considered a useful pilot study before a chronic trial, still representing the golden standard for confirming the effect observed in an acute trial. On the other hand, the increase in plasma NEAC detected in chronic studies suggests a positive association between endogenous and dietary antioxidants. In chronic

Table 4. Chronic supplementation studies with beverages and solid foods containing antioxidants and plasma non-enzymatic antioxidant capacity in human subjects: effect of methodology

(Number of interventions, participants and $95 \%$ confidence intervals)

\begin{tabular}{|c|c|c|c|c|c|c|c|}
\hline \multirow[b]{2}{*}{ Assays } & \multirow[b]{2}{*}{ Type of studies } & \multirow[b]{2}{*}{ No. of interventions } & \multirow[b]{2}{*}{ No. of participants } & \multirow[b]{2}{*}{ Results } & \multicolumn{2}{|c|}{ Effect size } & \multirow[b]{2}{*}{$P^{\star}$} \\
\hline & & & & & SMD & $95 \% \mathrm{Cl}$ & \\
\hline \multirow[t]{2}{*}{ TRAP } & Acute & 12 & 148 & $\uparrow$ & $1 \cdot 113$ & $0.486,1.741$ & 0.001 \\
\hline & Chronic & 15 & 269 & $\uparrow$ & 0.574 & $0.078,1.071$ & 0.023 \\
\hline \multirow{2}{*}{ FRAP } & Acute & 14 & 226 & $\uparrow$ & 1.059 & $0.612,1.505$ & $<0.001$ \\
\hline & Chronic & 23 & 674 & $\uparrow$ & 0.366 & $0.076,0.656$ & 0.013 \\
\hline \multirow[t]{2}{*}{ ORAC } & Acute & 11 & 115 & $\uparrow$ & 1.308 & $0.704,1.911$ & $<0.001$ \\
\hline & Chronic & 19 & 686 & $\uparrow$ & 0.653 & $0.304,1.001$ & 0.001 \\
\hline \multirow[t]{2}{*}{ TEAC } & Acute & 6 & 96 & $\leftrightarrow$ & 0.08 & $-0.660,0.819$ & 0.819 \\
\hline & Chronic & 21 & 707 & $\leftrightarrow$ & 0.318 & $-0.075,0.711$ & 0.113 \\
\hline
\end{tabular}

SMD, standardised mean difference; TRAP, total radical-trapping antioxidant parameter; $\uparrow$, increased; FRAP, ferric-reducing antioxidant potential; ORAC, oxygen radical absorbance capacity; TEAC, Trolox equivalent antioxidant capacity; $\leftrightarrow$, unchanged.

${ }^{*}$ Test for the overall effect. 
studies, we face a physiological mechanism of homeostatic control, aimed to tune an endogenous antioxidant network. More specifically, it might be possible that in a 'healthy condition' such as the absence of specific risk factors for oxidative stress, the body does not require an upload of dietary antioxidants. In order to test this hypothesis, we divided all chronic intervention studies on the basis of the 'healthy status' of the subjects.

Interestingly, and in agreement with previous evidence ${ }^{(7)}$, we observed a strong antioxidant response in the 'OSRRF' category that is three times higher than the 'healthy' category. In particular, plant-derived beverages (tea, fruit juices and red wine) are unable to increase plasma NEAC in healthy subjects, whereas plant-derived foods (chocolate, fruit, vegetables and dietary patterns based on plant-derived food) enhance plasma NEAC both in the healthy and OSRRF groups, with a double effect size in the last category. In this view, when chronic intervention studies were conducted on healthy subjects characterised by the lack of specific OSRRF, the body's antioxidant defences might better cope with free radical formation, preventing the need for dietary antioxidants. On the contrary, an increased intake of dietary antioxidant compounds could be identified as a helpful strategy to potentiate endogenous redox defences if pro-oxidant conditions are present.

In order to evaluate NEAC, several methodological approaches were performed so as to give birth to a major obstacle in understanding the pros and cons of NEAC as a biomarker for assessing antioxidant capacity in vivo. Based on hydrogen atom transfer (TRAP and ORAC) or singleelectron transfer (FRAP and TEAC), these methods could provide information related to the redox network in the plasma compartment after antioxidant ingestion ${ }^{(7,9)}$. Our data revealed that all methods, except TEAC, showed a similar trend following plant-derived food and beverage consumption. In particular, TRAP and ORAC displayed a double response in plasma NEAC in acute studies when compared with chronic studies, whereas the FRAP method showed a three times higher antioxidant response in acute studies than in chronic studies. On the contrary, the TEAC assay did not reveal any change in plasma NEAC neither in acute nor in chronic trials. The ORAC and TRAP assays measure the antioxidant inhibition of peroxyl radical-induced oxidation and therefore reflect classical radical chain-breaking antioxidant activity by hydrogen atom transfer. On the other hand, the TEAC assay is based on the ability of antioxidants in scavenging the radical cation 2,2'-azino-bis(3-ethylbenzothiazoline6-sulphonic acid). Biases of this method are related to plasma dilution and to non-physiological conditions of analysis that strongly reduce its sensitivity to detect plasma NEAC modulation in biological fluids. In fact, the peroxidase process does not mimic radical formation in vivo and the assay uses a non-physiological radical. Although the methods used revealed a good reproducibility in measuring plasma NEAC, among all the methods to detect the hydrogen atom transfer reaction, we suggest to use the TRAP and/or ORAC assays, whereas FRAP could be more sensitive than the TEAC assay (single-electron transfer-based methods) to assess plasma NEAC. Finally, the present results highlight the importance of assessing plasma non-enzymatic antioxidant defences through a battery of methodologies furnishing complementary information on different modalities of action of dietary antioxidants ${ }^{(22,23)}$.

Although several meta-analyses have shown a decreased risk of oxidative stress-related disease after vegetable food consumption $^{(24-26)}$, there are no meta-analyses measuring the impact of vegetable foods on plasma antioxidant markers in human subjects. In the present meta-analysis, we evaluated 122 intervention studies with 2890 analysed subjects, so that the precision and power of the analysis is increased. Moreover, about $86 \%$ of the included trials were performed with more than ten participants; this highlights the validity of the present results ${ }^{(6)}$.

However, the present meta-analysis has some limitations. First, the reviewed studies in the present meta-analysis are extremely heterogeneous, as they have different study designs. Indeed, in the meta-analysis, about $62.5 \%$ of the total intervention studies were not randomised and controlled, whereas about $46.6 \%$ of the included studies had a randomised and controlled design. Finally, about $37 \cdot 5 \%$ of all the intervention trials were randomised and controlled with a longitudinal study design.

Second, due to the difficulties in planning an appropriate placebo, clear evidence about the antioxidant potential of plant-derived foods and beverages is still far to come. However, on the basis of the above-described considerations, in order to evaluate the risk of overestimating intervention effects, all trials were stratified according to the study design (experimental and quasi-experimental design), and both EXP and Q-EXP studies have been processed together only after evaluating that their direction effects were similar ${ }^{(13)}$.

In conclusion, our data suggest that ingestion of plant foods and beverages increases plasma antioxidant potential in human subjects. We also showed that the antioxidant effect of plant foods is most conspicuous in subjects exposed to OSRRF, compared with those who are not exposed. These findings suggest the importance of considering exposure to oxidative stress as an important variable in deriving the benefits from the antioxidant action of plant foods. Accordingly, the present findings suggest the importance of introducing the concept of the 'redox necessity for a redox effectiveness' of antioxidants, beneficial when the body is under stress and not in all phases of life, if a plant food-based diet is routinely followed. Further chronic intervention studies involving subjects sharing a common need for dietary antioxidants are warranted to unravel the relative contributions of dietary and endogenous antioxidants.

\section{Supplementary material}

To view supplementary material for this article, please visit http://dx.doi.org/10.1017/S0007114513000263

\section{Acknowledgements}

D. L.-B. designed the study and directed its implementation, including quality assurance and control. F. T. contributed to 
the analysis of the data and revised the article. A. S. contributed to the interpretation of the data and to the writing and revising of the manuscript. G. M. conducted the literature review and contributed to the writing and revising of the manuscript. M. S. helped in supervising the project, designing the study's analytic strategy and writing and revising of the manuscript. No conflict of interest exists for each author. No funding was received.

\section{References}

1. Serafini M, Jakszyn P, Luján-Barroso L, et al. (2012) Dietary total antioxidant capacity and gastric cancer risk in the European prospective investigation into cancer and nutrition study. Int J Cancer 131, E544-E554.

2. Key TJ (2011) Fruit and vegetables and cancer risk. Br J Cancer 104, 6-11.

3. Khan N, Afaq F \& Mukhtar H (2008) Cancer chemoprevention through dietary antioxidants: progress and promise. Antioxid Redox Signal 10, 475-510.

4. Bjelakovic G, Nikolova D, Gluud LL, et al. (2008) Antioxidant supplements for prevention of mortality in healthy participants and patients with various diseases. The Cochrane Database of Systematic Reviews issue 2, CD007176.

5. Bjelakovic G, Gluud LL, Nikolova D, et al. (2011) Antioxidant supplements for liver diseases. The Cochrane Database of Systematic Reviews issue 3, CD007749.

6. Bjelakovic G, Nikolova D, Gluud LL, et al. (2007) Mortality in randomized trials of antioxidant supplements for primary and secondary prevention: systematic review and metaanalysis. JAMA 297, 842-857.

7. Serafini M, Villano D, Spera G, et al. (2006) Redox molecules and cancer prevention: the importance of understanding the role of the antioxidant network. Nutr Cancer 56, 232-240.

8. Serafini M, Miglio C, Peluso I, et al. (2011) Modulation of plasma non enzymatic antioxidant capacity (NEAC) by plant foods: the role of polyphenols. Curr Top Med Chem 11, 1821-1846.

9. Bartosz G (2010) Non-enzymatic antioxidant capacity assays: limitations of use in biomedicine. Free Radic Res 44, 711-720.

10. Barbato DL, Tomei G, Tomei F, et al. (2010) Traffic air pollution and oxidatively generated DNA damage: can urinary 8-oxo-7,8-dihydro-2-deoxiguanosine be considered a good biomarker? A meta-analysis. Biomarkers 15, 538-545.

11. Harris AD, Bradham DD, Baumgarten M, et al. (2004) The use and interpretation of quasi-experimental studies in infectious diseases. Clin Infect Dis 38, 1586-1591.

12. Harris AD, McGregor JC, Perencevich EN, et al. (2006) The use and interpretation of quasi-experimental studies in medical informatics. J Am Med Inform Assoc 13, 16-23.

13. Borenstein M, Hedges LV, Higgins JPT, et al. (2009) Introduction to Meta-analysis. Chichester: Wiley pp. 360-361.

14. Manchikanti L, Datta S, Smith HS, et al. (2009) Evidencebased medicine, systematic reviews, and guidelines in interventional pain management: part 6. Systematic reviews and meta-analyses of observational studies. Pain Physician 12, 819-850.

15. Higgins JPT (2008) Cochrane Handbook for Systematic Reviews of Interventions. Chichester: Wiley-Blackwell.

16. Deeks JJ, Higgins JPT, Altman DG (2008) Analysis data and undertaking meta-analysis. In Systematic Reviews of Inter- ventions, pp. 268-271 [JPT Higgins and S Green, editors]. Chichester: Wiley-Blackwell.

17. DerSimonian R \& Laird N (1986) Meta-analysis in clinical trials. Control Clin Trials 7, 177-188.

18. Higgins JP \& Thompson SG (2002) Quantifying heterogeneity in a meta-analysis. Stat Med 21, 1539-1558.

19. Sterne JA, Gavaghan D \& Egger M (2000) Publication and related bias in meta-analysis: power of statistical tests and prevalence in the literature. J Clin Epidemiol 53, 1119-1129.

20. Lim JS, Mietus-Snyder M, Valente A, et al. (2010) The role of fructose in the pathogenesis of NAFLD and the metabolic syndrome. Nat Rev Gastroenterol Hepatol 7, 251-264.

21. Fraga CG, Actis-Goretta L, Ottaviani JI, et al. (2005) Regular consumption of a flavanol-rich chocolate can improve oxidant stress in young soccer players. Clin Dev Immunol $\mathbf{1 2}$ $11-17$.

22. Lettieri-Barbato D, Villaño D, Beheydt B, et al. (2012) Effect of ingestion of dark chocolates with similar lipid composition and different cocoa content on antioxidant and lipid status in healthy humans. Food Chem 132, 1305-1310.

23. Villaño D, Lettieri-Barbato D, Guadagni F, et al. (2012) Effect of acute consumption of oolong tea on antioxidant parameters in healthy individuals. Food Chem $\mathbf{1 3 2}$ 2102-2106.

24. Desch S, Schmidt J, Kobler D, et al. (2010) Effect of cocoa products on blood pressure: systematic review and metaanalysis. Am J Hypertens 23, 97-103.

25. Zheng XX, Xu YL, Li SH, et al. (2011) Green tea intake lowers fasting serum total and LDL cholesterol in adults: a meta-analysis of 14 randomized controlled trials. Am J Clin Nutr 94, 601-610.

26. Aune D, Lau R, Chan DS, et al. (2011) Nonlinear reduction in risk for colorectal cancer by fruit and vegetable intake based on meta-analysis of prospective studies. Gastroenterology 141, 106-118.

27. Bertipaglia de Santana M, Mandarino MG, Cardoso JR, et al. (2008) Association between soy and green tea (Camellia sinensis) diminishes hypercholesterolemia and increases total plasma antioxidant potential in dyslipidemic subjects. Nutrition 24, 562-568.

28. Erba D, Riso P, Bordoni A, et al. (2005) Effectiveness of moderate green tea consumption on antioxidative status and plasma lipid profile in humans. J Nutr Biochem $\mathbf{1 6}$ 144-149.

29. Panza VS, Wazlawik E, Ricardo Schütz G, et al. (2008) Consumption of green tea favorably affects oxidative stress markers in weight-trained men. Nutrition 24, 433-442.

30. Coimbra S, Castro E, Rocha-Pereira P, et al. (2006) The effect of green tea in oxidative stress. Clin Nutr 25, 790-796.

31. van het Hof KH, de Boer HS, Wiseman SA, et al. (1997) Consumption of green or black tea does not increase resistance of low-density lipoprotein to oxidation in humans. Am J Clin Nutr 66, 1125-1132.

32. Hodgson JM, Puddey IB, Croft KD, et al. (2000) Acute effects of ingestion of black and green tea on lipoprotein oxidation. Am J Clin Nutr 71, 1103-1107.

33. Serafini M, Ghiselli A \& Ferro-Luzzi A (1996) In vivo antioxidant effect of green and black tea in man. Eur J Clin Nutr 50, 28-23.

34. Davies MJ, Judd JT, Baer DJ, et al. (2003) Black tea consumption reduces total and LDL cholesterol in mildly hypercholesterolemic adults. J Nutr 133, 3298S33025 . 
35. Maxwell S \& Thorpe G (1996) Tea flavonoids have little short term impact on serum antioxidant activity. BMJ 313, 229.

36. Duffy SJ, Keaney JF Jr, Holbrook M, et al. (2001) Short- and long-term black tea consumption reverses endothelial dysfunction in patients with coronary artery disease. Circulation 104, 151-156.

37. Kyle JA, Morrice PC, McNeill G, et al. (2007) Effects of infusion time and addition of milk on content and absorption of polyphenols from black tea. J Agric Food Chem 55 , 4889-4894.

38. Widlansky ME, Duffy SJ, Hamburg NM, et al. (2005) Effects of black tea consumption on plasma catechins and markers of oxidative stress and inflammation in patients with coronary artery disease. Free Radic Biol Med 38, 499-506.

39. Vukovic J, Modun D, Budimir D, et al. (2009) Acute, foodinduced moderate elevation of plasma uric acid protects against hyperoxia-induced oxidative stress and increase in arterial stiffness in healthy humans. Atherosclerosis 207, $255-260$.

40. Maxwell S, Cruickshank A \& Thorpe G (1994) Red wine and antioxidant activity in serum. Lancet 344, 193-194.

41. Modun D, Music I, Vukovic J, et al. (2008) The increase in human plasma antioxidant capacity after red wine consumption is due to both plasma urate and wine polyphenols. Atherosclerosis 197, 250-256.

42. Arendt BM, Ellinger S, Kekic K, et al. (2005) Single and repeated moderate consumption of native or dealcoholized red wine show different effects on antioxidant parameters in blood and DNA strand breaks in peripheral leukocytes in healthy volunteers: a randomized controlled trial (ISRCTN68505294). Nutr J 4, 33.

43. Serafini M, Maiani G \& Ferro-Luzzi A (1998) Alcohol-free red wine enhances plasma antioxidant capacity in humans. J Nutr 128, 1003-1007.

44. Avellone G, Di Garbo V, Campisi D, et al. (2006) Effects of moderate Sicilian red wine consumption on inflammatory biomarkers of atherosclerosis. Eur J Clin Nutr 60, 41-47.

45. Blackhurst DM \& Marais AD (2006) Concomitant consumption of red wine and polyunsaturated fatty acids in edible oil does not influence the peroxidation status of chylomicron lipids despite increasing plasma catechin concentration. Nutr Metab Cardiovasc Dis 16, 550-558.

46. Cao G, Booth SL, Sadowski JA, et al. (1998) Increases in human plasma antioxidant capacity after consumption of controlled diets high in fruit and vegetables. Am J Clin Nutr 68, 1081-1087.

47. Day A \& Stansbie D (1995) Cardioprotective effect of red wine may be mediated by urate. Clin Chem 41, 1319-1320.

48. Fernández-Pachón MS, Villaño D, Troncoso AM, et al. (2005) Antioxidant capacity of plasma after red wine intake in human volunteers. I Agric Food Chem 53 , 5024-5029.

49. Pinzani P, Petruzzi E, Magnolfi SU, et al. (2010) Red or white wine assumption and serum antioxidant capacity. Arch Gerontol Geriatr 51, 72-74.

50. Simonetti P, Gardana C \& Pietta P (2001) Plasma levels of caffeic acid and antioxidant status after red wine intake. J Agric Food Chem 49, 5964-5968.

51. Otaolaurruchi E, Fernández-Pachón MS, Gonzalez AG, et al. (2007) Repeated red wine consumption and changes on plasma antioxidant capacity and endogenous antioxidants (uric acid and protein thiol groups). J Agric Food Chem 55, 9713-9718.

52. Tsang C, Higgins S, Duthie GG, et al. (2005) The influence of moderate red wine consumption on antioxidant status and indices of oxidative stress associated with CHD in healthy volunteers. Br J Nutr 93, 233-240.

53. Guarda E, Godoy I, Foncea R, et al. (2005) Red wine reduces oxidative stress in patients with acute coronary syndrome. Int J Cardiol 104, 35-38.

54. Kiviniemi TO, Saraste A, Toikka JO, et al. (2007) A moderate dose of red wine, but not de-alcoholized red wine increases coronary flow reserve. Atherosclerosis 195, $176-181$

55. Visioli F, Riso P, Grande S, et al. (2003) Protective activity of tomato products on in vivo markers of lipid oxidation. EurJ Nutr 42, 201-206.

56. Tyssandier V, Feillet-Coudray C, Caris-Veyrat C, et al. (2004) Effect of tomato product consumption on the plasma status of antioxidant microconstituents and on the plasma total antioxidant capacity in healthy subjects. $\mathrm{J} \mathrm{Am}$ Coll Nutr 23, $148-145$.

57. Stracke BA, Rüfer CE, Bub A, et al. (2009) Bioavailability and nutritional effects of carotenoids from organically and conventionally produced carrots in healthy men. BrJ Nutr 101, 1664-1672.

58. Shen YC, Chen SL \& Wang CK (2007) Contribution of tomato phenolics to antioxidation and down-regulation of blood lipids. J Agric Food Chem 55, 6475-6481.

59. Serafini M, Bugianesi R, Salucci M, et al. (2002) Effect of acute ingestion of fresh and stored lettuce (Lactuca sativa) on plasma total antioxidant capacity and antioxidant levels in human subjects. Br J Nutr 88, 615-623.

60. Pellegrini N, Riso P \& Porrini M (2000) Tomato consumption does not affect the total antioxidant capacity of plasma. Nutrition 16, 268-271.

61. Lee A, Thurnham DI \& Chopra M (2000) Consumption of tomato products with olive oil but not sunflower oil increases the antioxidant activity of plasma. Free Radic Biol Med 29, 1051-1055.

62. Castenmiller JJ, West CE, Linssen JP, et al. (1999) The food matrix of spinach is a limiting factor in determining the bioavailability of beta-carotene and to a lesser extent of lutein in humans. J Nutr 129, 349-355.

63. Ahuja KD \& Ball MJ (2006) Effects of daily ingestion of chilli on serum lipoprotein oxidation in adult men and women. Br J Nutr 96, 239-242.

64. McAnlis GT, McEneny J, Pearce J, et al. (1999) Absorption and antioxidant effects of quercetin from onions, in man. Eur J Clin Nutr 53, 92-96.

65. Jacob K, Periago MJ, Böhm V, et al. (2008) Influence of lycopene and vitamin $\mathrm{C}$ from tomato juice on biomarkers of oxidative stress and inflammation. BrJ Nutr 99, 137-146.

66. Cao G, Russell RM, Lischner N, et al. (1998) Serum antioxidant capacity is increased by consumption of strawberries, spinach, red wine or vitamin C in elderly women. $J$ Nutr 128, 2383-2390.

67. Mathur S, Devaraj S, Grundy SM, et al. (2002) Cocoa products decrease low density lipoprotein oxidative susceptibility but do not affect biomarkers of inflammation in humans. J Nutr 132, 3663-3667.

68. Flammer AJ, Hermann F, Sudano I, et al. (2007) Dark chocolate improves coronary vasomotion and reduces platelet reactivity. Circulation 116, 2376-2382.

69. Serafini M, Bugianesi R, Maiani M, et al. (2003) Plasma antioxidants from chocolate. Nature 424, 1013.

70. Murphy KJ, Chronopoulos AK, Singh I, et al. (2003) Dietary flavanols and procyanidin oligomers from cocoa (Theobroma cacao) inhibit platelet function. Am J Clin Nutr 77, 1466-1473. 
71. Rein D, Lotito S, Holt RR, et al. (2000) Epicatechin in human plasma: in vivo determination and effect of chocolate consumption on plasma oxidation status. J Nutr 130, 8S Suppl., 2109S-2114S

72. Wiswedel I, Hirsch D, Kropf S, et al. (2004) Flavanol-rich cocoa drink lowers plasma F(2)-isoprostane concentrations in humans. Free Radic Biol Med 37, 411-421.

73. Mursu J, Voutilainen S, Nurmi T, et al. (2004) Dark chocolate consumption increases HDL cholesterol concentration and chocolate fatty acids may inhibit lipid peroxidation in healthy humans. Free Radic Biol Med 37, 1351-1359.

74. Engler MB, Engler MM, Chen CY, et al. (2004) Flavonoidrich dark chocolate improves endothelial function and increases plasma epicatechin concentrations in healthy adults. J Am Coll Nutr 23, 197-204.

75. Wang JF, Schramm DD, Holt RR, et al. (2000) A doseresponse effect from chocolate consumption on plasma epicatechin and oxidative damage. J Nutr 130, 8S Suppl., 2115S-2119S.

76. Vlachopoulos C, Aznaouridis K, Alexopoulos $\mathrm{N}$, et al. (2005) Effect of dark chocolate on arterial function in healthy individuals. Am J Hypertens 18, 785-791.

77. Roberts WG, Gordon MH \& Walker AF (2003) Effects of enhanced consumption of fruit and vegetables on plasma antioxidant status and oxidative resistance of LDL in smokers supplemented with fish oil. Eur J Clin Nutr 57, 1303-1310.

78. Urquiaga I, Strobel P, Perez D, et al. (2010) Mediterranean diet and red wine protect against oxidative damage in young volunteers. Atherosclerosis 211, 694-699.

79. Leighton F, Cuevas A, Guasch V, et al. (1999) Plasma polyphenols and antioxidants, oxidative DNA damage and endothelial function in a diet and wine intervention study in humans. Drugs Exp Clin Res 25, 133-141.

80. Razquin C, Martinez JA, Martinez-Gonzalez MA, et al. (2009) A 3 years follow-up of a Mediterranean diet rich in virgin olive oil is associated with high plasma antioxidant capacity and reduced body weight gain. Eur J Clin Nutr 63, 1387-1393.

81. Svendsen M, Blomhoff R, Holme I, et al. (2007) The effect of an increased intake of vegetables and fruit on weight loss, blood pressure and antioxidant defense in subjects with sleep related breathing disorders. Eur J Clin Nutr $\mathbf{6 1}$, $1301-1311$.

82. Miller ER 3rd, Erlinger TP, Sacks FM, et al. (2005) A dietary pattern that lowers oxidative stress increases antibodies to oxidized LDL: results from a randomized controlled feeding study. Atherosclerosis 183, 175-182.

83. Arendt BM, Boetzer AM, Lemoch $\mathrm{H}$, et al. (2001) Plasma antioxidant capacity of HIV-seropositive and healthy subjects during long-term ingestion of fruit juices or a fruitvegetable-concentrate containing antioxidant polyphenols. Eur J Clin Nutr 55, 786-792.

84. Castilla P, Echarri R, Dávalos A, et al. (2006) Concentrated red grape juice exerts antioxidant, hypolipidemic, and antiinflammatory effects in both hemodialysis patients and healthy subjects. Am J Clin Nutr 84, 252-262.

85. Bub A, Watzl B, Blockhaus $\mathrm{M}$, et al. (2003) Fruit juice consumption modulates antioxidative status, immune status and DNA damage. $J$ Nutr Biochem 14, 90-98.

86. Cilla A, De Palma G, Lagarda MJ, et al. (2009) Impact of fruit beverage consumption on the antioxidant status in healthy women. Ann Nutr Metab 54, 35-42.

87. Duthie SJ, Jenkinson AM, Crozier A, et al. (2006) The effects of cranberry juice consumption on antioxidant status and biomarkers relating to heart disease and cancer in healthy human volunteers. Eur J Nutr 45, 113-122.

88. Guo C, Wei J, Yang J, et al. (2008) Pomegranate juice is potentially better than apple juice in improving antioxidant function in elderly subjects. Nutr Res 28, 72-77.

89. O'Byrne DJ, Devaraj S, Grundy SM, et al. (2002) Comparison of the antioxidant effects of Concord grape juice flavonoids alpha-tocopherol on markers of oxidative stress in healthy adults. Am J Clin Nutr 76, 1367-1374.

90. Park YK, Lee SH, Park E, et al. (2009) Changes in antioxidant status, blood pressure, and lymphocyte DNA damage from grape juice supplementation. Ann NY Acad Sci 1171, 385-390.

91. Riso P, Visioli F, Gardana C, et al. (2005) Effects of blood orange juice intake on antioxidant bioavailability and on different markers related to oxidative stress. J Agric Food Chem 53, 941-947.

92. Young JF, Nielsen SE, Haraldsdóttir J, et al. (1999) Effect of fruit juice intake on urinary quercetin excretion and biomarkers of antioxidative status. Am J Clin Nutr 69 , 87-94.

93. Borochov-Neori H, Judeinstein S, Greenberg A, et al. (2006) Phenolic antioxidants and antiatherogenic effects of Marula (Sclerocarrya birrea subsp. caffra) fruit juice in healthy humans. J Agric Food Chem 56, 9884-9891.

94. Gorinstein S, Caspi A, Libman I, et al. (2004) Fresh Israeli jaffa sweetie juice consumption improves lipid metabolism and increases antioxidant capacity in hypercholesterolemic patients suffering from coronary artery disease: studies in vitro and in humans and positive changes in albumin and fibrinogen fractions. J Agric Food Chem $\mathbf{5 2}$ $5215-5222$.

95. Netzel M, Strass G, Kaul C, et al. (2002) In vivo antioxidative capacity of a composite berry juice. Food Res Intl 35, 213-216.

96. Netzel M, Strass G, Herbst M, et al. (2005) The excretion and biological antioxidant activity of elderberry antioxidants in healthy humans. Food Res Intl 38, 905-910.

97. Pedersen CB, Kyle J, Jenkinson AM, et al. (2000) Effects of blueberry and cranberry juice consumption on the plasma antioxidant capacity of healthy female volunteers. Eur $J$ Clin Nutr 54, 405-408

98. Prior RL, Gu L, Wu X, et al. (2007) Plasma antioxidant capacity changes following a meal as a measure of the ability of a food to alter in vivo antioxidant status. $\mathrm{J} \mathrm{Am} \mathrm{Coll}$ Nutr 26, 170-181.

99. Ruel G, Pomerleau S, Couture P, et al. (2005) Changes in plasma antioxidant capacity and oxidized low-density lipoprotein levels in men after short-term cranberry juice consumption. Metabolism 54, 856-861.

100. Vinson JA, Bose P, Proch J, et al. (2008) Cranberries and cranberry products: powerful in vitro, ex vivo, and in vivo sources of antioxidants. J Agric Food Chem $\mathbf{5 6}$ 5884-5891.

101. Wilms LC, Boots AW, de Boer VC, et al. (2007) Impact of multiple genetic polymorphisms on effects of a 4 -week blueberry juice intervention on ex vivo induced lymphocytic DNA damage in human volunteers. Carcinogenesis $\mathbf{2 8}$, 1800-1806.

102. McAnulty SR, McAnulty LS, Morrow JD, et al. (2005) Effect of daily fruit ingestion on angiotensin converting enzyme activity, blood pressure, and oxidative stress in chronic smokers. Free Radic Res 39, 1241-1248.

103. Chaves AA, Joshi MS, Coyle CM, et al. (2009) Vasoprotective endothelial effects of a standardized grape product in humans. Vascul Pharmacol 50, 20-26. 
104. Lotito SB \& Frei B (2004) Relevance of apple polyphenols as antioxidants in human plasma: contrasting in vitro and in vivo effects. Free Radic Biol Med 36, 201-211.

105. Mazza G, Kay CD, Cottrell T, et al. (2002) Absorption of anthocyanins from blueberries and serum antioxidant status in human subjects. J Agric Food Chem 50, 7731-7737.

106. Parker TL, Wang XH, Pazmiño J, et al. (2007) Antioxidant capacity and phenolic content of grapes, sun-dried raisins, and golden raisins and their effect on ex vivo serum antioxidant capacity. J Agric Food Chem 55, 8472-8477.

107. Rankin JW, Andreae MC, Oliver Chen CY, et al. (2008) Effect of raisin consumption on oxidative stress and inflammation in obesity. Diabetes Obes Metab $\mathbf{1 0}$ 1086-1096.

108. Serafini M, Testa MF, Villaño D, et al. (2009) Antioxidant activity of blueberry fruit is impaired by association with milk. Free Radic Biol Med 46, 769-774.

109. Tesoriere L, Butera D, Pintaudi AM, et al. (2004) Supplementation with cactus pear (Opuntia ficus-indica) fruit decreases oxidative stress in healthy humans: a comparative study with vitamin C. Am J Clin Nutr 80, $391-395$

110. Vinson JA, Zubik L, Bose P, et al. (2005) Dried fruits: excellent in vitro and in vivo antioxidants. I Am Coll Nutr 24, 44-50. 